\title{
EDITORIAL
}

\section{Entre pequeña empresa familiar y gran familia de empresas: una muestra de los grupos empresariales en América Latina desde la perspectiva de la historia empresarial}

Los grupos empresariales en América Latina han desatado un renovado interés en las últimas décadas, especialmente porque desde el inicio de la segunda fase de globalización se convirtieron en los líderes de la internacionalización de las empresas de la región (Andonova \& Losada-Otálora, 2017; Bull et al., 2014; Casanova, 2010), han estado en el ojo del huracán en muchos países por su estrecha relación con la política y el Estado y, contrario a lo observado en países desarrollados, se fortalecieron y crecieron aceleradamente. Diferente a lo ocurrido durante los años 70, cuando los trabajos publicados sobre grupos demonizaban su existencia (Cordero, 1977; Dahse, 1979; Silva-Colmenares, 1977), historiadores económicos y empresariales concluyen que los grupos son uno de los mayores actores económicos en la región, junto con las multinacionales y las empresas propiedad del Estado (Miller, 2010). Lo anterior ha permitido la publicación de estudios sobre la evolución de grupos individuales, descripciones de las características de los grupos en algunos países y estudios cuantitativos sobre el impacto de los grupos en la economía o la internacionalización (Barbero, 2021), lo cual ha generado un renovado interés por su evolución en los diferentes períodos de la historia económica de la región.

La pregunta aún latente en la literatura sobre grupos empresariales en países emergentes es aquella relacionada con la razón por la cual los individuos, las familias o los empresarios regionales propietarios de empresas (pymes y grandes) tienden a aumentar sus inversiones en diferentes sectores y a organizarse como grupos empresariales que persisten en el tiempo (Barbero \& Puig, 2016). Las discusiones sobre los orígenes, la consolidación y la persistencia de los grupos alrededor del mundo les han asignado una relativa importancia en las economías emergentes (Colpan 
\& Hikino, 2010, 2018; Khanna \& Yafeh, 2007), que combinada con la identificación de la flexibilidad de su estructura para adaptarse al contexto (Bull et al., 2014; Geymonat, 2020; Rodriguez-Satizabal, 2020a, 2020b) y la persistencia de la propiedad familiar (Cerutti, 2006, 2015; Fernández \& Lluch, 2015), nos lleva a preguntarnos por la evolución histórica de las estrategias de las familias y sus grupos en economías en constante transformación, como las latinoamericanas.

Considerando el creciente interés en estudiar con perspectiva histórica la reacción de la empresa a cambios en los mercados donde se encuentra, y entendiendo que los grupos empresariales son la estructura de crecimiento predilecta de las familias empresariales, y en algunos casos de las empresas propiedad del Estado, este dossier explora el vínculo entre la persistencia de los grupos empresariales y sus estrategias de diversificación (producto y geográfica) e internacionalización ante entornos cambiantes. Teniendo en cuenta la estrecha relación entre la historia empresarial y la literatura en estrategia (Casson \& Da-Silva-Lopes, 2013), este dossier reproduce el contexto histórico en el que tienen lugar los grupos empresariales de Argentina, Brasil, México y El Salvador durante un período que se expande desde 1827 hasta 2019. Los artículos aquí publicados buscan aportar a la comprensión de las complejas dimensiones temporales a través de las cuales se toman decisiones sobre la estrategia tanto de las empresas afiliadas como del grupo.

En este dossier se entienden los grupos empresariales (Colpan \& Hikino, 2010) como un conjunto de empresas independientes, legalmente constituidas, que operan en diferentes industrias (relacionadas y no relacionadas) y están unidas por vínculos formales (por ejemplo, estructura de capital) e informales (por ejemplo, propiedad familiar). No son empresas agrupadas por alianzas de corto plazo y no están constituidas en una sola entidad, pero son propiedad del mismo dueño (empresario, familia o grupo de empresarios), y muchas veces controladas por este. Sin embargo, se consideran 
también a las familias, las elites empresariales y la empresa familiar (Lluch \& Fernández, 2021) como unidades de análisis conducentes a la creación de redes de propiedad, como lo son los grupos.

Las preguntas que direccionaron la convocatoria de este dossier incluyeron ¿cuál es la relación entre los cambios en el contexto económico y político y las estrategias de los grupos?, ¿cuál es la relación entre los eventos en la familia propietaria y las estrategias de los grupos?, ¿qué empresas afiliadas se benefician más con las decisiones de estrategia?, ¿se premia a aquellas en la actividad económica principal, a las más grandes, a las nuevas?, ¿cómo se financiaron los grupos?, ¿cuál ha sido su relación con la banca comercial y los mercados de capitales?, ¿cuáles fueron las principales fuentes y usos del capital?, ¿cómo y por qué se formaron mercados internos (capital, recursos, gerenciales)?, ¿qué mercados internos (capital, recursos, gerenciales) se fortalecieron o debilitaron en uno u otro período?, ¿por qué y cómo los grupos adaptan su portafolio de productos a cambios en el modelo económico?, ¿por qué y cómo se internacionalizan los grupos?, ¿qué redes de propiedad y control crean los grupos?

Las respuestas de los ocho investigadores, seis hombres y dos mujeres, desde diferentes perspectivas, como la historia, la economía y la administración, nos ha permitido publicar un conjunto de seis artículos que incluyen tres casos de estudio sobre la evolución de un grupo de un país específico - dos de Brasil, uno de Argentina-, un estudio sobre una elite de inmigrantes en México, un trabajo sobre una empresa estadounidense que formó una red de propiedad en Brasil y una investigación que caracteriza a los grupos de El Salvador. En todos los trabajos, los autores procuraron presentar la evolución histórica de las estrategias de las empresas y los grupos con base en archivos institucionales y trabajos previos. La metodología elegida es la narrativa histórica y la presentación de estadísticas básicas que permiten entrever las estrategias adoptadas frente a cambios en el contexto 
político, diversificación de producto, inserción a nuevos mercados domésticos e internacionalización.

Importante señalar en este punto tres detalles del dossier relacionados con el tamaño de las economías de la región, el diálogo entre comunidades académicas y el fortalecimiento de la historia empresarial en Centroamérica. Siguiendo con la tendencia en la literatura de historia económica y empresarial, cinco de los trabajos se concentran en tres de los países más grandes de la región, Argentina, Brasil y México, que además tienen las familias y grupos empresariales de mayor trayectoria y tamaño en América Latina; además de constituir ejemplos de empresas que apostaron por convertirse en multilatinas. Respondiendo al llamado de la importancia del diálogo entre los investigadores latinoamericanos, este dossier publica tres trabajos en español y tres en portugués, abriendo un espacio para que quienes investigamos sobre familias y grupos empresariales iniciemos una polinización cruzada que permita caracterizar y analizar mejor la trayectoria de estas unidades de estudio. Apoyando el espacio generado por la historia empresarial de América Central, el dossier incluye un artículo sobre un país en donde la evolución del empresariado se encuentra en la mira de historiadores, economistas y administradores, quienes están abriendo caminos para nuevas generaciones de investigadores.

Sin embargo, el dossier peca por no incluir trabajos sobre Colombia, Perú, Uruguay y Chile, países donde hay un grupo de investigadores estudiando familias y grupos empresariales con metodologías que incluyen tipologías, análisis de redes, recolección de indicadores de desempeño, una mirada a la relación entre familia y estrategia desde la perspectiva de la teoría organizacional y una indagación sobre la relación con las fuentes de financiación. Estos temas son algunos de los que marcarán las futuras investigaciones sobre la región y que esperamos sean motivo de otro dossier. Su otra debilidad es la baja respuesta a la pregunta sobre las estrategias de financiación de las familias, grupos y empresas afiliadas, asunto 
que necesita mayor atención por parte de quienes estudiamos la región, puesto que al entender las fuentes y los usos que las redes de propiedad le dan al capital, podríamos indagar más sobre las razones de las estrategias y la consolidación de los grupos, entender el tardío desarrollo de nuestros mercados de capitales, la evolución de la banca y su relación con el empresariado, profundizar en el papel del gobierno y establecer variables que nos permitan dimensionar el rol de los empresarios en el desarrollo económico de nuestros países.

Los tres casos de estudio hacen referencia a grandes grupos en sus respectivos países. El trabajo de Susana Bandieri (Conicet e IPEHCS) sobre la familia Braun-Menéndez Behety presenta la evolución de La Anónima S. A. a grupo empresarial desde finales del siglo XIX, con un énfasis en la estrategia de diversificación promovida desde las primeras generaciones de la familia. Su trabajo incluye una nueva perspectiva sobre el empresariado regional que suma a las discusiones sobre el rol de las familias tradicionales en regiones específicas de nuestros países. El estudio de Gustavo Pereira (Universidade Federal de São Carlos) y Armando Dalla Costa (Universidade Federal do Paraná) sobre el Grupo Votorantim resalta por la cantidad de fuentes revisadas y el buen uso que les dan a ellas para explicar el proceso de internacionalización de esta gran multilatina. Este trabajo se concentra en el período de 1980 a 2019, años con fuertes crisis, y se refugia en los cambios en el contexto económico para presentar las reacciones estratégicas del grupo. Así mimo, este se destaca por el uso de indicadores de desempeño para discutir las formas de financiación de Votorantim y responde a la pregunta con respecto al uso de diferentes fuentes de capital por parte de los grupos para sobrevivir y gatillar la internacionalización. En el último caso, lan Kisil Marino (Universidade Estadual de Campinas) y Pedro Giovannetti Moura (Universidade de São Paulo) se arriesgan a entrar en el ojo del huracán de Odebrecht, combinando la historia del grupo con su estrategia de responsabilidad social. Este artículo debate la tensión entre las medidas neoliberales, la expansión internacional y 
el interés de mantener una relación justa y equilibrada con las comunidades de los países que los reciben.

Los tres trabajos restantes presentan redes de propiedad. José Galindo (Universidad Veracruzana) analiza las estrategias empresariales de los barcelonettes —elite de empresarios inmigrantes - en México, vinculando las generaciones de sus familias con las decisiones de diversificación de producto, las redes sociales y de negocios que formaron, y la importancia otorgada a la cohesión del capital familiar. El trabajo hace el ejercicio de identificar los cambios en las decisiones de negocio generación tras generación, indicando los ajustes en las inversiones y las relaciones. Alan dos Santos Ribeiro (Universidade Federal Fluminense) presenta el caso de la expansión en Brasil de la empresa estadounidense de propiedad familiar Maxwell, Wright \& Co., durante el período 1827-1850. Este artículo presenta el crecimiento de la empresa y el fortalecimiento de la propiedad en manos de dos familias muy conectadas con la actividad transatlántica, lo cual fue posible gracias a las inversiones de este grupo en subsectores del comercio agrícola y de esclavos. Por último, Lissette Canales (Universidad Tecnológica de El Salvador) presenta la historia abreviada de 21 grupos empresariales de El Salvador, con un énfasis especial en la relación con la política, el Estado y la banca. Este es un trabajo que aporta indicadores de la relación de los grupos con la política, al identificar la contribución de estos a campañas políticas y la puerta giratoria de sus presidentes.

Finalmente, este dossier incluye dos reseñas de libros publicados en 2021. Julio César Zuluaga (Universidad Javeriana de Cali) hace una revisión del libro "Historia empresarial en América Latina: temas, debates y problemas", editado por Andrea Lluch, Martin Monsalve y Marcelo Bucheli. Por su parte, Sonia Jaimes (Universidad ICESI) hace una revisión del libro "Fabricato 100 años. La tela de los hilos perfectos", coordinado por Karim León Vargas y Juan Carlos López Díez (Universidad EAFIT). El primer libro es un hito en 
la historia empresarial de la región. El segundo es la historia de un grupo empresarial colombiano.

Este dossier es un aporte a la literatura existente sobre familias y grupos empresariales que deseamos continúe creciendo. Esperamos que esta iniciativa permita más espacios para el análisis comparativo, la discusión teórica y la identificación de estrategias de formación y consolidación de redes de propiedad en la región.

\section{Beatriz Rodríguez-Satizabal \\ Profesora de la Universidad del Pacífico, Perú bh.rodriguezs@up.edu.pe https://orcid.org/0000-0003-1872-5520 https://doi.org/10.21789/24222704.1778}

\section{REFERENCIAS}

Andonova, V., \& Losada-Otálora, M. (2017). Multilatinas: Strategies for internationalisation. Cambridge University Press.

https://doi.org/DOI:10.1017/9781316417706

Barbero, M. I. (2021). Los grupos económicos latinoamericanos. En A. Lluch, M. Monsalve, \& M. Bucheli (eds.), Historia empresarial en América Latina: temas, debates y problemas. Universidad del Pacífico y Universidad de los Andes.

Barbero, M. I., \& Puig, N. (2016). Business groups around the world: An introduction. Business History, 58(1), 6-29.

https://doi.org/10.1080/00076791.2015.1051530 
Bull, B., Castellacci, F., \& Kasahara, Y. (2014). Business groups and transnational capitalism in Central America. Economic and political strategies (1. ${ }^{\text {a }}$ ed.). Palgrave Macmillan UK. https://doi.org/10.1057/9781137359407

Casanova, L. (2010). Las multinacionales latinoamericanas en los albores de una gran oportunidad. Revista de Administraçao de Empresas, 50(4). https://doi.org/10.1590/S0034-75902010000400008

Casson, M., \& Da-Silva-Lopes, T. (2013). Foreign direct investment in highrisk environments a historical perspective. Business History, 55(3), 375-404. https://doi.org/10.1080/00076791.2013.771343

Cerutti, M. (2006). Empresas y grupos empresariales en América Latina, España y Portugal. Universidad Autónoma de Nuevo León \& Universidad de Alicante.

Cerutti, M. (2015). Grandes empresas y familias empresariales en México. En P. Fernández \& A. Lluch (eds.), Familias empresarias y grandes empresas familiares en América Latina y España. Una visión de largo plazo (pp. 153-188). Fundación BBVA.

Colpan, A. M., \& Hikino, T. (2010). Foundations of business groups: Towards an integrated framework. En A. M. Colpan, T. Hikino, \& J. R. Lincoln (eds.), The Oxford handbook of business groups (pp. 15-66). Oxford University Press. https://doi.org/10.1093/oxfordhb/9780199552863.003.0002

Colpan, A. M., \& Hikino, T. (eds.). (2018). Business groups in the West: Origins, evolution, and resilience. Oxford University Press. https://doi.org/10.1093/oso/9780198717973.001.0001

Cordero, S. (1977). Concentración industrial y poder económico en México (vol. 18). Cuadernos del CES, El Colegio de México.

Dahse, F. (1979). Mapa de la extrema riqueza. Los grupos económicos y el proceso de concentración de capitales. Editorial Aconcagua. 
Fernández, P., \& Lluch, A. (eds.). (2015). Familias empresarias y grandes empresas familiares en América Latina y España. Una visión de largo plazo. Fundación BBVA.

Geymonat, J. (2020). Industrial business groups in a context of deindustrialization. The Uruguayan case, 1980-2015. Journal of Evolutionary Studies in Business, 5(2), 150-176.

https://doi.org/10.1344/jesb2020.2.j079

Khanna, T., \& Yafeh, Y. (2007). Business groups in emerging markets: paragons or parasites? Journal of Economic Literature, 45(2), 331-372. https://doi.org/10.1257/jel.45.2.331

Lluch, A., \& Fernández, P. (2021). Familias empresarias y empresas familiares en América Latina. En A. Lluch, M. Monsalve, \& M. Bucheli (eds.), Historia empresarial en América Latina: temas, debates y problemas. Universidad del Pacífico y Universidad de los Andes.

Miller, R. (2010). Latin American business history and varieties of capitalism. Business History Review, 84(4), 653-657. https://doi.org/www.jstor.org/stable/27917302

Rodriguez-Satizabal, B. (2020a). Only one way to raise capital? Colombian business groups and the dawn of internal markets. Business History, 1-22. https://doi.org/10.1080/00076791.2020.1796973

Rodriguez-Satizabal, B. (2020b). Pathways from deglobalisation: Colombian business groups, 1950-1985. Journal of Evolutionary Studies in Business, 5(2), 177-214. https://doi.org/10.1344/jesb2020.2.j080

Silva-Colmenares, J. (1977). Los verdaderos dueños del país. Fondo Editorial Suramérica. 